BULL. AUSTRAL. MATH. SOC.

\title{
ON THE BOUNDARY BEHAVIOUR OF BLOCH AND NORMAL FUNCTIONS
}

\section{RAUNO AULASKARI}

Criteria for an analytic function $f$ defined in $|z|<1$ to belong to $B_{0}$, the class of Bloch functions satisfying $\lim _{|z| \rightarrow 1}\left(1-|z|^{2}\right)\left|f^{\prime}(z)\right|=0$, and criteria for a meromorphic function $g$ defined in $|z|<1$ to belong to $N_{O}$, namely, to satisfy $\lim _{|z| \rightarrow 1}\left(1-|z|^{2}\right) \frac{\left|g^{\prime}(z)\right|}{1+|g(z)|^{2}}=0$, are obtained in terms of the area and the length of the images of hyperbolic disks and hyperbolic circles, respectively.

$\$ 1$.

Let $f$ be a holomorphic function in the unit disk $D=\{z|| z \mid<1\}$ of the complex plane $\ell=\{z|| z \mid<\infty\}$. Let $B$ be the family of holomorphic functions $f$ in $D$ such that

$$
\sup _{z \in D}\left(1-|z|^{2}\right)\left|f^{\prime}(z)\right|<\infty
$$

and $B_{O}$ the family of holomorphic functions $f$ in $D$ such that

$$
\lim _{|z| \rightarrow 1}\left(1-|z|^{2}\right)\left|f^{\prime}(z)\right|=0
$$

If $f \in B$, then $f$ is said to be a Bloch function. In Theorem 1 we shall propose some criteria for $f$ to belong to ${ }^{B} O_{0}$. These criteria are

Received 13 June 1984.

Copyright Clearance Centre, Inc. Serial-fee code: 0004-9727/84 $\$ A 2.00+0.00$ 
immediate consequences of Yamashita's Theorem in [4].

Let

$$
d(z, w)=\frac{1}{2} \log \frac{|1-z \bar{w}|+|z-w|}{|1-z \bar{w}|-|z-w|}
$$

be the hyperbolic distance between $z$ and $w$ in $D$. For $0<r<\infty$ and for $z \in D$, we set

$$
U(z, r)=\{w \in D \mid d(w, z)<r\}
$$

and

$$
\Gamma(z, r)=\{w \in D \mid d(w, z)=r\}
$$

Let $A_{f}(z, r)$ be the euclidean area of the Riemannian image $F(z, r)$ of $U(z, r)$ by $f$, and let $A_{f}(z, r)$ be the euclidean area of the image $F(z, r)$ of $U(z, r)$ by $f$; we note that $F(z, r)$ is the projection of $F(z, r)$ to C. Let $L_{f}(z, r)$ be the euclidean length of the Riemannian image of $\Gamma(z, r)$ by $f_{f}$ and $L_{f}(z, r)$ the euclidean length of the outer boundary of $F(z, r)$. The outer boundary of a bounded domain $G$ in $\not$ means the boundary of $\mathscr{C} \backslash E$, where $E$ is the unbounded component of the complement $\phi \backslash G$ of $G$. The inequalities

$$
A_{f}(z, r) \geq A_{f}(z, r) \text { and } L_{f}(z, r) \geqq L_{f}(z, r)
$$

hold for each $0<r<\infty$ and each $z \in D$.

Yamashita proved the following:

THEOREM A. Let $f$ be non-constant and holomorphic in $D$. Then the following are mutually equivalent:

(I) $f \in B$;

(II) there exists $0<r<\infty$ such that $\sup _{z \in D} A_{f}(z, r)<\infty$;

(III) there exists $0<r<\infty$ such that $\sup _{z \in D} A_{f}(z, r)<\infty$;

(IV) there exists $0<r<\infty$ such that $\sup _{z \in D} L_{f}(z, r)<\infty$; 
(V) there exists $0<r<\infty$ such that $\sup _{z \in D} L_{f}(z, r)<\infty$.

From this theorem we obtain

THEOREM 1. Let $f$ be non-constant and holomorphic in $D$. Then the following are mutually equivalent:

(I) $f \in B_{0}$;

(II) there exists $0<r<\infty$ such that $\lim _{|z| \rightarrow 1} A_{f}(z, r)=0$;

(III) there exists $0<r<\infty$ such that $\lim _{|z| \rightarrow 1} A_{f}(z, r)=0$;

(IV) there exists $0<r<\infty$ such that $\lim _{|z| \rightarrow 1} L_{f}(z, r)=0$;

(V) there exists $0<r<\infty$ such that $\underset{|z| \rightarrow 1}{\lim } L_{f}(z, r)=0$.

Proof. The assertions follow immediately from the proof of Theorem $A$ in [4] by replacind the bounded term by a sequence of terms converging to zero.

$\S 2$.

The meromorphic analogue of a Bloch function is a normal meromorphic function. A function $f$, meromorphic in $D$, is said to be normal in $D$ if $\sup _{\left(1-|z|^{2}\right) f^{*}(z)<\infty}$ where $f^{*}(z)=\left|f^{\prime}(z)\right| /\left(1+|f(z)|^{2}\right)$ is the $z \in D$

spherical derivative of $f$ (cf. [3]). We denote by $N$ the family of all normal meromorphic functions in $D$. Further, let $N_{0}$ be the family of meromorphic functions $f$ in $D$ such that

$$
\lim _{|z| \rightarrow 1}\left(1-|z|^{2}\right) f^{*}(z)=0
$$

The euclidean area and length used in the above theorems will be replaced by the spherical area and spherical length. We shall

denote the spherical area of $F(z, r)$ by $B_{f}(z, r)$ and the spherical area of $F(z, r)$ by $B_{f}(z, r)$. Let $M_{f}(z, r)$ be the spherical length of the 
Riemannian image of $\Gamma(z, r)$ by $f$, and let $M_{f}(z, r)$ be the length of the boundary of $E(z, r)$. The corresponding inequalities as above are valid, that is,

$$
B_{f}(z, r) \geqq B_{f}(z, r) \text { and } M_{f}(z, r) \geqq M_{f}(z, r)
$$

For normal meromorphic functions we cannot obtain results corresponding to those in Theorem $A$, as shown by Yamashita in [4]. For example, implication (III) $\Rightarrow(I)$ does not hold as Lappan has shown in [2] and the implication $(V) \Rightarrow(I)$ is still open. Therefore it is interesting to notice that the meromorphic analogue of Theorem 1 for the functions of $N_{0}$ is valid. For the proof of our theorem we shall make use of the following lemma [1, Lemma II] :

LEMMA. For the function $g$ meromorphic in $D$ suppose that the spherical area $B_{g}(O, r)$ is strictly less than $\pi$. Then,

$$
g^{*}(0)^{2} \leqq \frac{B_{g}(0, r)}{\pi x^{2}\left(1-\frac{B_{g}(0, r)}{\pi}\right)},
$$

where $x=\left(e^{2 r}-1\right) /\left(e^{2 r}+1\right)$.

THEOREM 2. Let $f$ be non-constant and meromorphic in $D$. Then the following are mutually equivalent:

(I) $\quad f \in N_{0}$;

(II) there exists $0<r<\infty$ such that $\lim _{|z| \rightarrow 1} B_{f}(z, r)=0$;

(III) there exists $0<r<\infty$ such that $\underset{|z| \rightarrow 1}{\lim B} B_{f}(z, r)=0$;

(IV) there exists $0<r<\infty$ such that $\lim _{|z| \rightarrow 1} M_{f}(z, r)=0$ and $B_{f}(z, r) \leqq \alpha<\pi$ for alz $z, r_{0}<|z|<1$;

(V) there exists $0<r<\infty$ such that $\lim _{|z| \rightarrow 1} M_{f}(z, r)=0$ and $B_{f}(z, r) \leqq \alpha<\pi$ for all $z, r_{0}<|z|<1$. 
Proof. We prove first $(I I I) \Rightarrow(I)$; let

$$
g(w)=f\left(\frac{w+z}{1+\bar{z} w}\right)
$$

By the assumption there is a $r_{0}>0$ such that $B_{f}(z, r)<\pi$ for all $z, r_{0}<|z|<1$. Let $|z|>r_{0}$. Then by a simple calculation and the Lemma we have

$$
\begin{aligned}
\left(1-|z|^{2}\right) f^{*}(z) & =g^{*}(0) \leqq\left\{\frac{B g(0, r)}{\pi x^{2}\left(1-\frac{B g(0, r)}{\pi}\right)}\right\}^{1 / 2} \\
& =\left\{\frac{B f(z, r)}{\pi x^{2}\left(1-\frac{B_{f}^{(z, r)}}{\pi}\right.}\right\}^{1 / 2},
\end{aligned}
$$

where $x=\left(e^{2 r}-1\right) /\left(e^{2 r}+1\right)$. Hence $(I I I) \Rightarrow(I) . \quad(I) \Leftrightarrow(I I)$

Yamashita has proved this result in [5]. (II) $\Rightarrow$ (IV) By the above equivalence it is sufficient to prove that $(I) \Rightarrow(I V)$. We choose a sequence of points $\left(z_{n}\right)$ for which $\left|z_{n}\right| \rightarrow 1$ as $n \rightarrow \infty$. Let $r>0$. We take the sequence of hyperbolic disks $\left(U\left(z_{n}, r\right)\right)$ and form the functions

$$
f_{n}(\zeta)=f\left\{\frac{\zeta+z_{n}}{1+\bar{z}_{n} \zeta}\right\} .
$$

Let $\zeta_{0} \in \Gamma(0, r)$ and let $z_{n}^{\prime}=\left(\zeta_{0}+z_{n}\right) /\left(1+\bar{z}_{n} \zeta_{0}\right)$. The radius of $D$ going through $z_{n}$ intersects $\Gamma\left(z_{n}, r\right)$ in two points. We denote by $z_{n}^{\prime \prime}$ the point for which $\left|z_{n}^{\prime \prime}\right|<\left|z_{n}\right|$. Then we obtain for the spherical derivative

$$
\begin{gathered}
f_{n}^{*}\left(5_{0}\right)=\frac{1}{1-\delta\left(z_{n}, z_{n}^{\prime}\right)^{2}} \cdot\left(1-\left|z_{n}^{\prime}\right|^{2}\right) f^{*}\left(z_{n}^{\prime}\right) \\
\leqq \frac{1}{\alpha}\left(1-\left|z_{n}^{\prime \prime}\right|^{2}\right) \max _{z \in \Gamma\left(z_{n}, r\right)} f^{*}(z)=\frac{1}{\alpha}\left(1-\left|z_{n}^{\prime \prime}\right|^{2}\right) f^{*}\left(z_{n}^{\prime \prime \prime}\right),
\end{gathered}
$$


where $z_{n}^{\prime \prime \prime} \in \Gamma\left(z_{n}, r\right)$ and $1-\delta\left(z_{n}, z_{n}^{\prime}\right)^{2}=1-\left|\frac{z_{n}-z_{n}^{\prime}}{1-\bar{z}_{n}^{\prime}{ }_{n}}\right|^{2} \geqq \alpha>0$, since $d\left(z_{n}, z_{n}^{\prime}\right)=d\left(0, \zeta_{0}\right)=r . \quad$ Now

$$
\begin{aligned}
& M_{f}\left(z_{n}, r\right)=\underset{\Gamma\left(z_{n}, r\right)}{\int f^{*}(z)|d z|}=\int_{\Gamma(0, r)} f_{n}^{*}(\zeta)|d \zeta| \\
& \leqq \frac{1}{\alpha}\left(1-\left|z_{n}^{\prime \prime}\right|^{2}\right) f^{*}\left(z_{n}^{\prime \prime \prime}\right) \iint_{\Gamma(0, r)}|d \zeta| \\
& =\frac{\pi}{\alpha} \log \frac{1+r}{1-r} \cdot \frac{1-\left|z_{n}^{\prime \prime}\right|^{2}}{1-\left|z_{n}^{\prime \prime \prime}\right|^{2}}\left(1-\left|z_{n}^{\prime \prime \prime}\right|^{2}\right) f^{*}\left(z_{n}^{\prime \prime \prime}\right) \rightarrow 0,
\end{aligned}
$$

since $\left|z_{n}^{\prime \prime \prime}\right| \rightarrow 1$ and $\frac{1-\left|z_{n}^{\prime \prime}\right|^{2}}{1-\left|z_{n}^{\prime \prime}\right|^{2}}+1$

The latter part of the assertion follows from the assumption(II). $(I V) \Rightarrow(V)$ : This follows trivially from $(1) .(V) \Rightarrow(I I I)$ : Let $\left(z_{n}\right)$ be any sequence of points for which $\left|z_{n}\right| \rightarrow 1$ as $n \rightarrow \infty$. Then, for sufficiently large $n$, either the diameter of $F\left(z_{n}, r\right)$

$$
\operatorname{diam} F\left(z_{n}, r\right) \leqq M_{f}\left(z_{n}, r\right)
$$

or the complement $\hat{\mathscr{l}} \backslash F\left(z_{n}, r\right)$ is divided into the components $E_{i}\left(z_{n}, r\right), i \in I \quad(I$ an index set) for which

$$
\sum_{i \in I} \operatorname{diam} E_{i}\left(z_{n}, r\right) \leqq M_{f}\left(z_{n}, r\right)
$$

When $n$ is large enough, the latter alternative is not possible by the assumption $B_{f}(z, r) \leqq \alpha<\pi$. The assertion follows by (2) and thus the theorem is proved.

\section{References}

[1] J. Dufresnoy, "Sur 1'aire sphêrique décrite par les valeurs d'une fonction méromorphe", BulZ. Sci. Math. 65 (1941), 214-219. 
[2] P. Lappan, "A non-normal locally uniformly univalent function", BulZ. London Math. Soc. 5 (1973), 291-294.

[3] 0. Lehto and K. I. Virtanen, "Boundary behaviour and normal meromorphic functions", Acta Math. 97 (1957), 47-65.

[4] S. Yamashita, "Criteria for functions to be Bloch", Bull. Austral. Math. Soc. 21 (1980), 223-229.

[5] S. Yamashita, "Functions of uniformly bounded characteristic", Ann. Acad. Sci. Fenn. Ser. A I Math. 7 (1982), 349-367.

Department of Mathematics,

University of Joensuu,

P.0. Box 111 ,

SF-80101 Joensuu 10,

Finland. 